\title{
Effect of exercise training on lipid profiles in the elderly: A systematic review and meta-analysis of randomized controlled trials
}

\author{
Mahdi Ghafari $^{\mathbf{1}^{*}}$, Mohamad Faramarzi $^{\mathbf{2}^{\circledR}}$, Timothy Baghurst $^{3^{\circledR}}$ \\ 'Department of Sport Sciences, Shahrekord University, Shahrekord, Iran \\ ${ }^{2}$ Professor in Exercise Physiology, Faculty of Sport Sciences, Department of Exercise Physiology, University of Isfahan, Isfahan, \\ Iran \\ ${ }^{3}$ College of Education, Florida State University, Tallahassee, Florida, USA
}

*Corresponding Author: Mahdi Ghafari, Department of Sport Sciences, Shahrekord University, Shahrekord, Iran, Tel: +9893832324401, Email: Ghafari.mehdi@sku.ac.ir, Ghafari.mehdi@gmail.com

\begin{abstract}
Background and aims: There are many articles about the effects of different training methods on lipid profiles in the elderly. The objective of the present study was to investigate the influence of training on the lipid profile in the elderly.

Methods: In this study, databases of PubMed, Embase, Scopus, ScienceDirect, Web of Science, SID, Magiran, and Google Scholar were searched. Intervention effects were presented as mean difference (MD) with a random-effects model. Subgroup analysis and sensitivity analysis were performed to study heterogeneity, following the primary screening of the full text of the articles.

Results: A total of 23 trials with 1654 individuals were included in the meta-analysis. Levels of high-density lipoprotein cholesterol (HDL-C) $(\mathrm{MD}=0.47 \mathrm{mg} / \mathrm{dL} ; P<0.001, \mathrm{SE}=0.08, \mathrm{~V}=0.01,95 \% \mathrm{Cl}=0.310 .63, \mathrm{Z}=5.73,19$ trials), low-density lipoprotein cholesterol (LDL-C) $(\mathrm{MD}=-0.46 ; P<0.001, \mathrm{SE}=0.11, \mathrm{~V}=0.01,95 \% \mathrm{Cl}=-0.68-0.25, \mathrm{Z}=-4.24)$, triglyceride $(\mathrm{MD}=-0.62 ; P=0.001, \mathrm{SE}=0.12, \mathrm{~V}=0.01$, $95 \% \mathrm{Cl}=-0.86-0.38, Z=-5.03,20$ trials), and total cholesterol $(\mathrm{TC})(\mathrm{MD}=-0.33 ; P<0.001, \mathrm{SE}=0.09, \mathrm{~V}=0.01,95 \% \mathrm{Cl}=-0.52-0.15$, $Z=-3.57,16$ trials) were investigated. Following sensitivity analysis and heterogeneity testing, the results were still strong and impressive. Conclusion: Lipid profiles improved in training groups, indicating higher levels of HDL-C and lower levels of LDL-C, TC, and triglyceride. Overall, training leads to a better lipid profile. However, closer scrutiny seems necessary.

Keywords: Training, Triglyceride, Total cholesterol, HDL, LDL
\end{abstract}

Received: 7 March 2020, Accepted: 30 May 2020, ePublished: 29 December 2020

\section{Introduction}

Interventions among older individuals have concentrated on cardiovascular disease (CVD) biomarkers (1). Training has been approved as a therapeutic measure with an important role in cardiac health and preventive medicine $(2,3)$. Regular aerobic exercise appears to improve myocardial performance (3). Although normally ascribed to dyslipidemic profile and atherosclerosis (4), myocardial dysfunction has been reported in the absence of increased serum lipids (5). Because there is a relationship between myocardial disease and oxidative stress with dyslipidemic profile (5).

Lipids and lipoproteins as risk factors for CVDs in the elderly increase with age (1). Changes in circulating CVD markers are good indicators of the ideal impacts of training on cardiovascular health (6). A few examinations have indicated that the grown-ups with low degrees of high-density lipoprotein cholesterol (HDL-C) have a high risk of CVDs (7) and low-density lipoprotein cholesterol (LDL-C) was significantly correlated with CVDs and mortality (8). Observational studies comparing inactive and active groups confirm that training optimally decreases blood lipids (9). In some studies, ultra-endurance athletes had lower concentrations of LDL-C, higher concentrations of HDL-C, total cholesterol (TC), and triglycerides (TG) compared with a matched group (10).

Some reviews of the effect of training on the blood lipid have been published $(11,12)$. One meta-analysis (13) reported decreases in LDL-C, TG, and TC with increases in HDLC after training, other detailed reviews summarized different results. In this meta-analysis, different kinds of training with different intensity and duration were selected to evaluate the effect of training on lipid profile.

Therefore, considering 1) the lower than optimal prevalence of lipids and lipoproteins in the elderly 2) contradictory results of previous studies on the effect of lipids and lipoproteins in older people, and 3) lack of meta-analysis studies on the effect of training on lipids and lipoproteins in older people, this study was conducted to investigate the effect of training in on lipid profile in the elderly and provide recommendations for future research.

Materials and Methods

Literature search

(C) 2020 The Author(s); Published by Shahrekord University of Medical Sciences. This is an open-access article distributed under the terms of the Creative Commons Attribution License (http://creativecommons.org/licenses/by/4.0), which permits unrestricted use, distribution, and reproduction in any medium, provided the original work is properly cited. 
Databases of PubMed, Embase, Scopus, ScienceDirect, Web of Science, SID, Magiran, and Google Scholar were searched. In literature searches of Cochrane Controlled Trials Registry, the following keywords were searched: "[ exercise OR training OR physical activity OR Training] AND blood lipid OR blood fats OR lipedema OR Dyslipidemia OR hyperlipidemia OR hyperlipoproteinemia OR total cholesterol OR triglyceride OR high density lipoprotein cholesterol OR low-density lipoprotein cholesterol. Randomized controlled trials were included (RCTs) and compared with each type of the supervised training in terms of the outcome and reported means (or differences between means). Exclusion criteria were as follows: 1) RCTs that did not report enough data to complete meta-analysis; 2) Double-publications or subgroup analysis of trials.

\section{Data extraction}

Two examiners separately extracted all data. For the variables of interest, we extracted baseline, post-intervention mean and standard deviation, and sample sizes for intervention and control groups, following the Cochrane review protocols (14). Additional data obtained from abstracts included participants' characteristics (gender, age, and duration of diabetes), year of publication, dietary intervention, follow-up time, and training characteristics (duration, type, severity, and frequency) (15).

\section{Quality evaluation}

Two authors individually assessed the methodological quality of the trials. The Cochrane risk of bias tool was used to assess the risk of bias (16): allocation concealment (selection bias), random sequence generation (selection bias), blinding of outcome assessment (detection bias), blinding of participants and personnel (performance bias), incomplete outcome data (attrition bias), selective reporting (reporting bias), and other sources of bias. The experiments involved in the study had the following characteristics: random sequence generation (bias of selection), concealment allocation (bias of selection), blindness of participants and personnel (bias of performance), blindness of outcome assessment (bias of detection), incomplete outcome data (bias or apparent bias), selective reporting (bias of reporting) and other biases.

\section{Statistical analysis}

The analysis was completed using random-effect models $(16,17)$, and the consequences are shown as the weighted standard mean difference (SMD). The effect size of the intervention of each study was estimated in parameters between the control and training groups during the intervention. Heterogeneity was evaluated using $\mathrm{I}^{2}$ index (14). Variables that may affect the association between training and outcomes included gender, kinds of training (resistance, aerobic, or a combination of both), mean age, sessions per week, period of study (continuous, weeks) (14). The funnel plot was used to assess the publication bias and the bias was statistically measured by the Egger methods (16). Trim-and-fill method was used to estimate the potential impact of unpublished studies on our results (16). All analyses were conducted by STATA version 12.0 (Stata, College Station, TX, USA).

\section{Results}

Twenty-three studies with 1654 participants were included in the analysis, 871 subjects in the training groups and 783 subjects in the control groups. Figure 1 indicates an overview of the search plan. Six studies were conducted on women and two studies on men and fifteen studies on both genders. The age of the participants ranged from 50 to 83 years. The number of sessions per week ranged from 3 to 5 . The duration of interventions ranged from 7 to 52 weeks (Table 1).

\section{Influence of training on $H D L$}

The present meta-analysis shows that HDL levels significantly decreased by Training (Figure 2$)(\mathrm{MD}=0.47$ $\mathrm{mg} / \mathrm{dL} ; P<0.001, \mathrm{SE}=0.08, \mathrm{~V}=0.01,95 \% \mathrm{CI}=0.31$ $0.63, \mathrm{Z}=5.73,19$ trials), but the heterogeneity of the study remained significant $\left(\mathrm{I}^{2}=57 \%\right)$. In Figure 2 , aerobic training significantly increased HDL levels $(\mathrm{MD}=0.47$ $\mathrm{mg} / \mathrm{dL} ; P<0.001, \mathrm{SE}=0.15, \mathrm{~V}=0.02,95 \% \mathrm{CI}=0.18$ $0.76, Z=3.2, I^{2}=53 \%$ ), and resistance training significantly altered HDL levels $(\mathrm{MD}=0.84 ; P<0.001, \mathrm{SE}=0.24, \mathrm{~V}=$ $0.06,95 \% \mathrm{CI}=0.371 .31, \mathrm{Z}=3.52, \mathrm{I}^{2}=36 \%$ )

\section{Influence of training on $L D L$}

The present meta-analysis shows that LDL levels significantly decreased by training (Figure 3$)(\mathrm{MD}=-0.3$; $\mathrm{P}<0.001, \mathrm{SE}=0.08, \mathrm{~V}=0.01,95 \% \mathrm{CI}=-0.45-0.14$, $\mathrm{Z}=-3.81)$, but the heterogeneity of the study remained significant $(\mathrm{I} 2=68.8 \%)$. In subgroups, aerobic training significantly decreased LDL levels $(\mathrm{MD}=-0.49 ; P=0.001$, $\mathrm{SE}=0.15, \mathrm{~V}=0.02,95 \% \mathrm{CI}=-0.78-0.21, \mathrm{Z}=-3.36$, $\mathrm{I}^{2}=72 \%$ ), and resistance training significantly altered LDL levels $(\mathrm{MD}=-0.23 ; P=0.19, \mathrm{SE}=0.18, \mathrm{~V}=0.03,95 \% \mathrm{CI}$ $\left.=-0.590 .12, \mathrm{Z}=-1.31, \mathrm{I}^{2}=0 \%\right)$.

\section{Influence of Training on TC}

The present meta-analysis shows that TC levels significantly decreased by training (Figure 4) $(\mathrm{MD}=-0.24 ; P<0.001$, $\mathrm{SE}=0.07, \mathrm{~V}=0.01,95 \% \mathrm{CI}=-0.38-0.09, \mathrm{Z}=-3.23)$, and heterogeneity of the study was $33 \%$. In subgroups, aerobic training significantly decreased TG levels $(\mathrm{MD}=$ $-0.35 ; P=0, \mathrm{SE}=0.1, \mathrm{~V}=0.01,95 \% \mathrm{CI}=-0.55-0.15$, $\mathrm{Z}=-3.38, \mathrm{I}^{2}=52 \%$ ), and resistance training significantly altered TG levels $(\mathrm{MD}=-0.33 ; P=-1.22, \mathrm{SE}=0.27, \mathrm{~V}=$ $0.07,95 \% \mathrm{CI}=-0.850 .2, \mathrm{Z}=-1.22, \mathrm{I}^{2}=52 \%$ ) 


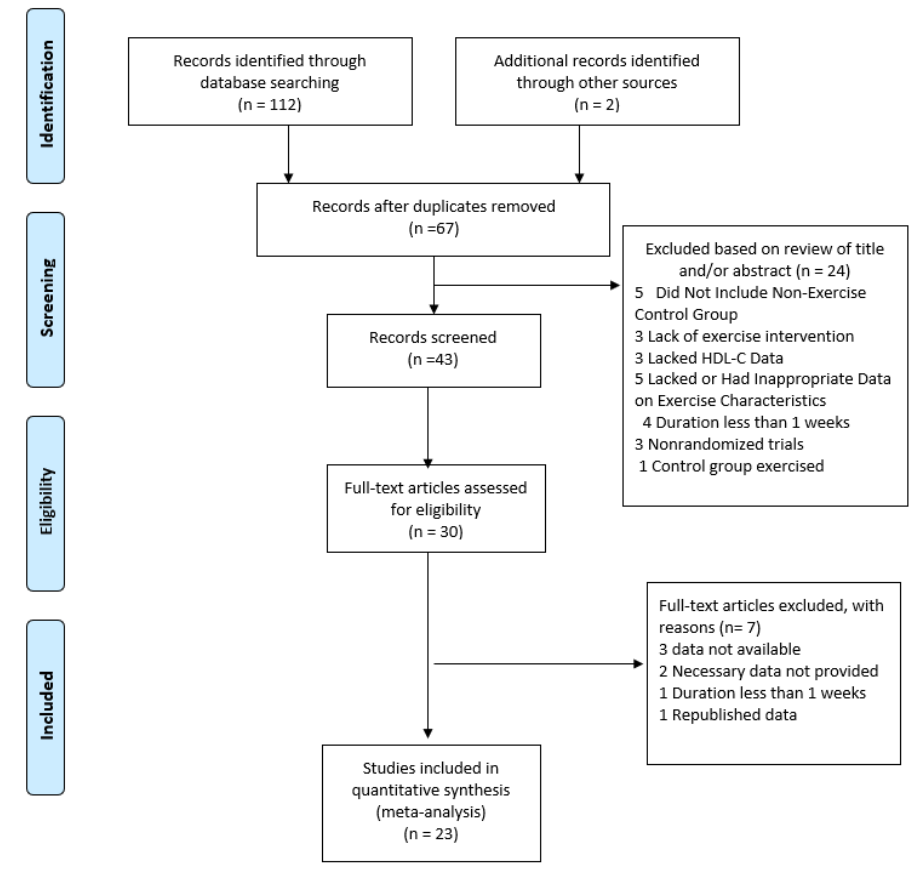

Figure 1. Flow chart of the study selection procedure.
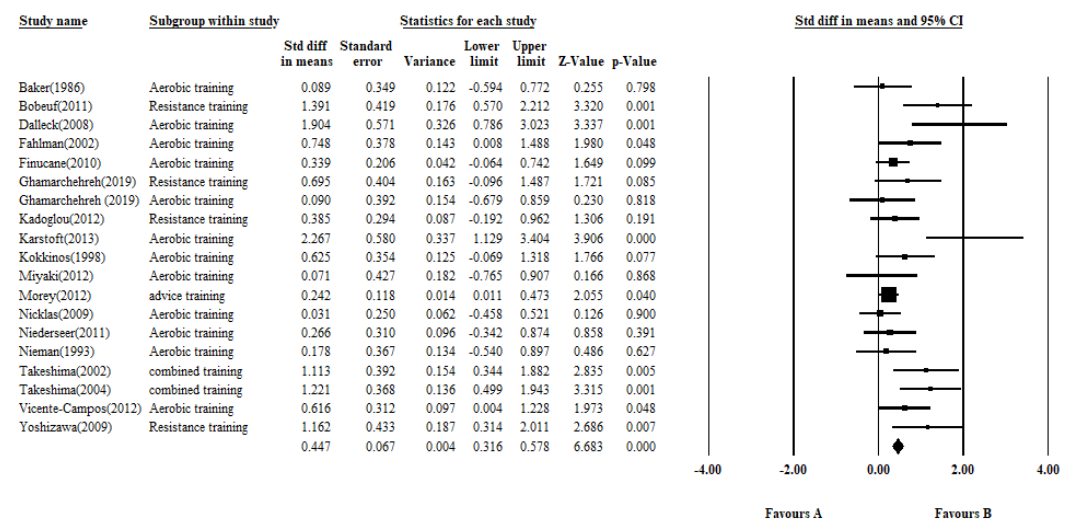

Figure 2. Effects of training on $\mathrm{HDL}(\mathrm{mg} / \mathrm{dL})$, forest plot showing pooled mean differences with $95 \% \mathrm{Cl}$ for 19 effect sizes obtained from 20 trials (two distinct effect sizes for various modalities were prepared by Ghamarchehreh et al) (30) .
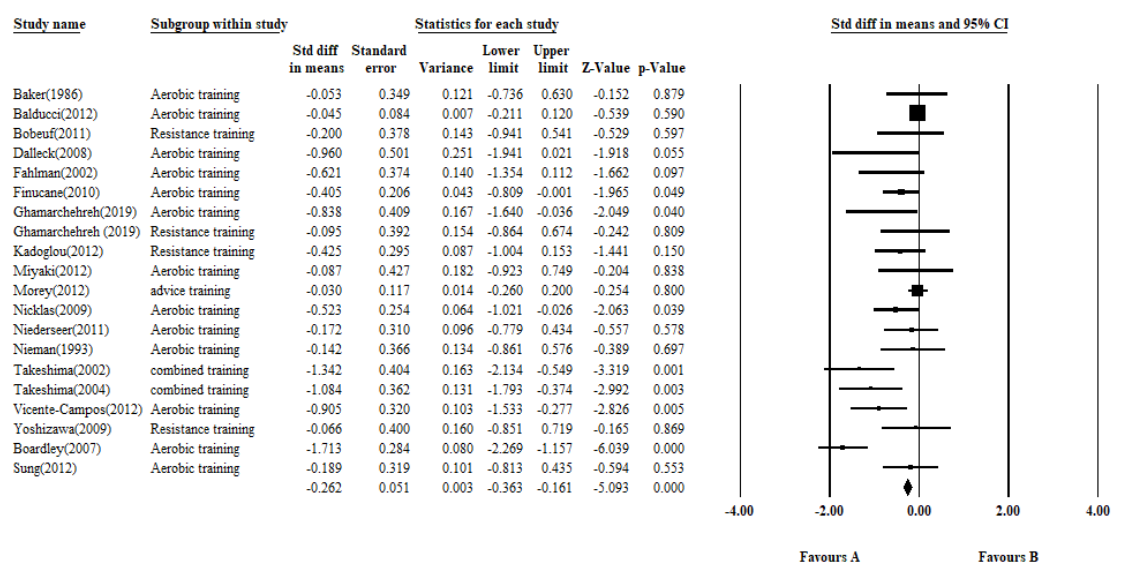

Figure 3. Effects of training on LDL ( $\mathrm{mg} / \mathrm{dL}$ ), forest plot showings pooled mean differences with $95 \%$ Cls for 19 effect sizes obtained from 20 trials (two distinct effect sizes for various modalities were prepared by Ghamarchehreh et al) (30). 


\begin{tabular}{|c|c|c|c|c|c|c|c|c|}
\hline \multirow[t]{2}{*}{ Study name } & \multicolumn{3}{|c|}{$\underline{\text { Subgroup within study }}$} & \multicolumn{5}{|c|}{$\underline{\text { Statistics for each study }}$} \\
\hline & & $\begin{array}{l}\text { Std diff } \\
\text { in means }\end{array}$ & $\begin{array}{l}\text { Standard } \\
\text { error }\end{array}$ & Variance & $\begin{array}{c}\text { Lower } \\
\text { limit }\end{array}$ & $\begin{array}{c}\text { Upper } \\
\text { limit }\end{array}$ & Z-Value & p-Val \\
\hline Baker(1986) & Aerobic training & -0.107 & 0.349 & 0.122 & -0.791 & 0.576 & -0.308 & 0.75 \\
\hline Bobeuf(2011) & Resistance trainin & -0.048 & 0.377 & 0.142 & -0.787 & 0.691 & -0.127 & 0.899 \\
\hline Fahlman(2002) & Aerobic training & -0.621 & 0.374 & 0.140 & -1.354 & 0.112 & -1.662 & 0.097 \\
\hline Finucane(2010) & Aerobic training & -0.405 & 0.206 & 0.043 & -0.809 & -0.001 & -1.965 & 0.049 \\
\hline Ghamarchehreh(2019) & Aerobic training & -0.358 & 0.395 & 0.156 & -1.133 & 0.417 & -0.906 & 0.365 \\
\hline Ghamarchehreh (2019) & Resistance training & -0.015 & 0.392 & 0.154 & -0.784 & 0.754 & -0.038 & 0.970 \\
\hline $\operatorname{Kadoglou}(2012)$ & Resistance training & -0.142 & 0.292 & 0.085 & -0.714 & 0.431 & -0.484 & 0.628 \\
\hline Miyaki(2012) & Aerobic training & -0.178 & 0.427 & 0.183 & -1.016 & 0.659 & -0.418 & 0.676 \\
\hline Morey(2012) & advice training & -0.015 & 0.117 & 0.014 & -0.245 & 0.215 & -0.126 & 0.900 \\
\hline Nicklas(2009) & Aerobic training & -0.444 & 0.253 & 0.064 & -0.939 & 0.051 & -1.758 & 0.07 \\
\hline Niederseer(2011) & Aerobic training & -0.120 & 0.309 & 0.096 & -0.726 & 0.486 & -0.388 & 0.69 \\
\hline Nieman(1993) & Aerobic training & -0.028 & 0.366 & 0.134 & -0.745 & 0.690 & -0.076 & 0.939 \\
\hline Takeshima(2002) & combined training & -1.342 & 0.404 & 0.163 & -2.134 & -0.549 & -3.319 & 0.001 \\
\hline Takeshima(2004) & combined training & -0.540 & 0.344 & 0.119 & -1.215 & 0.135 & -1.568 & 0.11 \\
\hline Vicente-Campos(2012) & Aerobic training & -0.616 & 0.312 & 0.097 & -1.228 & -0.004 & -1.973 & 0.04 \\
\hline Yoshizawa(2009) & Resistance training & -1.280 & 0.439 & 0.193 & -2.141 & -0.419 & -2.914 & 0.00 \\
\hline & & -0.334 & 0.094 & 0.009 & -0.518 & -0.151 & -3.569 & \\
\hline
\end{tabular}

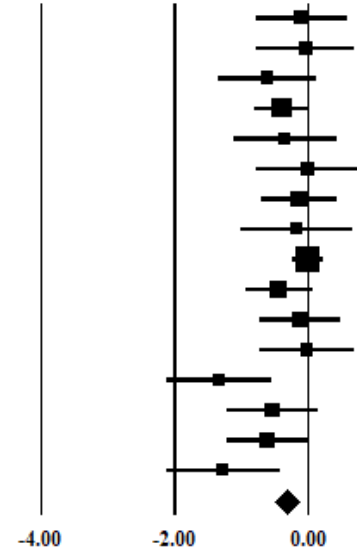

Favours A

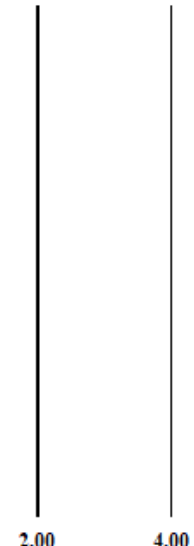

Favours B

Figure 4. Effects of Training on TC (mg/dL), forest plot showings pooled mean differences with $95 \%$ Cls for 15 effect sizes obtained from 16 trials (two distinct effect sizes for various modalities were prepared by Ghamarchehreh et al) (30).

\begin{tabular}{|c|c|c|c|c|c|c|c|c|}
\hline \multirow[t]{2}{*}{ Study name } & \multicolumn{3}{|l|}{ Subgroup within study } & \multicolumn{5}{|c|}{ Statistics for each study } \\
\hline & & $\begin{array}{c}\text { Std diff } \\
\text { in means }\end{array}$ & $\begin{array}{l}\text { Standard } \\
\text { error }\end{array}$ & Variance & $\begin{array}{l}\text { Lower } \\
\text { limit }\end{array}$ & $\begin{array}{c}\text { Upper } \\
\text { limit }\end{array}$ & Z-Value & p-Value \\
\hline Baker(1986) & Aerobic training & -0.075 & 0.349 & 0.122 & -0.759 & 0.608 & -0.216 & 0.829 \\
\hline Bobeuf(2011) & Resistance training & -0.147 & 0.378 & 0.143 & -0.887 & 0.593 & -0.389 & 0.697 \\
\hline Dalleck(2008) & Aerobic training & -2.435 & 0.624 & 0.390 & -3.659 & -1.212 & -3.901 & 0.000 \\
\hline Fahlman(2002) & Aerobic training & -0.621 & 0.374 & 0.140 & -1.354 & 0.112 & -1.662 & 0.097 \\
\hline Finucane(2010) & Aerobic training & -0.339 & 0.206 & 0.042 & -0.742 & 0.064 & -1.649 & 0.099 \\
\hline Ghamarchehreh(2019) & Aerobic training & -1.360 & 0.435 & 0.189 & -2.213 & -0.507 & -3.124 & 0.002 \\
\hline Ghamarchehreh (2019) & Resistance training & -0.494 & 0.398 & 0.159 & -1.274 & 0.286 & -1.241 & 0.215 \\
\hline Kadoglou(2012) & Resistance training & -0.086 & 0.292 & 0.085 & -0.658 & 0.486 & -0.294 & 0.769 \\
\hline Karstoft(2013) & Aerobic training & -2.267 & 0.580 & 0.337 & -3.404 & -1.129 & -3.906 & 0.000 \\
\hline Kokkinos(1998) & Aerobic training & -0.671 & 0.355 & 0.126 & -1.366 & 0.025 & -1.890 & 0.059 \\
\hline Miyaki(2012) & Aerobic training & -0.505 & 0.433 & 0.188 & -1.354 & 0.344 & -1.166 & 0.243 \\
\hline Morey(2012) & advice training & -0.079 & 0.117 & 0.014 & -0.309 & 0.151 & -0.675 & 0.500 \\
\hline Nicklas(2009) & Aerobic training & -0.323 & 0.251 & 0.063 & -0.815 & 0.169 & -1.287 & 0.198 \\
\hline Niederseer(2011) & Aerobic training & -1.022 & 0.328 & 0.108 & -1.665 & -0.378 & -3.111 & 0.002 \\
\hline Nieman(1993) & Aerobic training & -0.320 & 0.368 & 0.136 & -1.041 & 0.402 & -0.868 & 0.386 \\
\hline Takeshima(2002) & combined training & -1.342 & 0.404 & 0.163 & -2.134 & -0.549 & -3.319 & 0.001 \\
\hline Takeshima(2004) & combined training & -0.064 & 0.338 & 0.114 & -0.727 & 0.599 & -0.191 & 0.849 \\
\hline Vicente-Campos(2012) & Aerobic training & -0.616 & 0.312 & 0.097 & -1.228 & -0.004 & -1.973 & 0.048 \\
\hline Yoshizawa(2009) & Resistance training & -1.001 & 0.425 & 0.180 & -1.833 & -0.169 & -2.357 & 0.018 \\
\hline Boardley(2007) & Aerobic training & -1.285 & 0.266 & 0.071 & -1.807 & -0.762 & -4.820 & 0.000 \\
\hline Sung(2012) & Aerobic training & -0.013 & 0.318 & 0.101 & -0.636 & 0.610 & -0.041 & 0.967 \\
\hline & & -0.615 & 0.122 & 0.015 & -0.855 & -0.376 & -5.029 & 0.000 \\
\hline
\end{tabular}

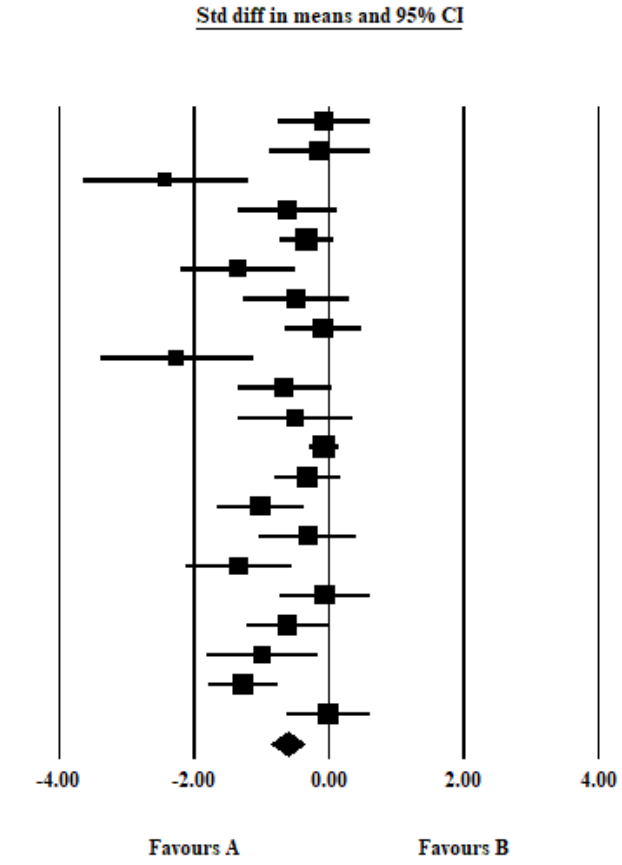

Figure 5. Effects of training on TG (mg/dL), forest plot showings pooled mean differences with $95 \%$ CIs for 20 effect sizes obtained from 21 trials (two distinct effect sizes for various modalities were prepared by Ghamarchehreh et al) (30). 
Table 1. Characteristics of the Included Studies

\begin{tabular}{|c|c|c|c|c|c|c|c|c|}
\hline Study & $\begin{array}{l}\text { Age }(y) \text { (mean or } \\
\text { rang) }\end{array}$ & $\begin{array}{l}\text { BMI, } \mathrm{kg} / \\
\mathrm{m}^{2}(\text { mean or } \\
\text { rang) }\end{array}$ & Gender & Marker & $\begin{array}{l}\text { Activity/medical } \\
\text { condition }\end{array}$ & $\begin{array}{l}\text { Duration } \\
\text { (wk) }\end{array}$ & NT/NC & $\begin{array}{l}\text { Exercise type, intensity and } \\
\text { cointerventions }\end{array}$ \\
\hline $\begin{array}{l}\text { Yoshizawa et al } \\
\text { (18) }\end{array}$ & $(50-65)$ & 23.7 & Female only & $\begin{array}{l}\text { TC, TG, HDL-C, } \\
\text { LDL-C }\end{array}$ & Sedentary/healthy & 8 & $12 / 13$ & $\begin{array}{l}\text { Resistance training/moderate/ } \\
\text { No }\end{array}$ \\
\hline $\begin{array}{l}\text { Vicente-Campos } \\
(2012)\end{array}$ & $(62-67)$ & $N R$ & $\begin{array}{l}\text { Male and } \\
\text { female }\end{array}$ & $\begin{array}{l}\text { TC, TG, HDL-C, } \\
\text { LDL-C }\end{array}$ & Sedentary/healthy & 28 & $22 / 21$ & Aerobic training/vigorous/No \\
\hline $\begin{array}{l}\text { Takeshima et al } \\
\text { (19) }\end{array}$ & $(60-83)$ & NR & $\begin{array}{l}\text { Male and } \\
\text { female }\end{array}$ & $\begin{array}{l}\text { TC, TG, HDL-C, } \\
\text { LDL-C }\end{array}$ & Sedentary/healthy & 12 & $18 / 17$ & $\begin{array}{l}\text { Progressive accommodating } \\
\text { circuit exercise/vigorous/No }\end{array}$ \\
\hline $\begin{array}{l}\text { Takeshima et al } \\
\text { (20) }\end{array}$ & $(60-75)$ & NR & $\begin{array}{l}\text { Male and } \\
\text { female }\end{array}$ & $\begin{array}{l}\text { TC, TG, HDL-C, } \\
\text { LDL-C }\end{array}$ & Sedentary/healthy & 7 & $15 / 15$ & $\begin{array}{l}\text { Endurance training (walking- } \\
\text { dancing, } 30 \mathrm{~min} \text { ), stretching, } \\
\text { resistance training/vigorous- No }\end{array}$ \\
\hline Nieman et al (21) & $(67-85)$ & 23.7 & $\begin{array}{l}\text { Male and } \\
\text { female }\end{array}$ & $\begin{array}{l}\text { TC, TG, HDL-C, } \\
\text { LDL-C, }\end{array}$ & Sedentary/healthy & 12 & $14 / 16$ & Walk/moderate/No \\
\hline $\begin{array}{l}\text { Niederseer et } \\
\text { al }(22)\end{array}$ & $\begin{array}{c}(\mathrm{C}: 67.3 \pm 4.4, \mathrm{~T}: \\
66.6 \pm 2.1)\end{array}$ & $\begin{array}{l}(\mathrm{T}: 27.1+3.3 \\
\mathrm{C}: 25.4+2.8)\end{array}$ & Female & $\begin{array}{l}\text { TC, TG, HDL-C, } \\
\text { LDL-C, CRP }\end{array}$ & Active/healthy & 12 & $22 / 20$ & Skiing/moderate/No \\
\hline Nicklas et al (23) & $(50-70)$ & 25 to 40 & $\begin{array}{l}\text { Male and } \\
\text { female }\end{array}$ & $\begin{array}{l}\text { TG, HDL-C, } \\
\text { LDL-C }\end{array}$ & Sedentary/healthy & 20 & $36 / 29$ & $\begin{array}{l}\text { Calorie restriction and aerobic } \\
\text { exercise /moderate/ }\end{array}$ \\
\hline Morgan et al (24) & $(50-70)$ & $N R$ & $\begin{array}{l}\text { Male and } \\
\text { female }\end{array}$ & TC, HDL-C & Sedentary/healthy & 15 & $14 / 15$ & Walk/moderate/No \\
\hline Morey et al (25) & $(60-89)$ & 25 to 45 & $\begin{array}{l}\text { Male and } \\
\text { female }\end{array}$ & $\begin{array}{l}\text { TG, HDL C, LDL } \\
\text { C, TC, fasting } \\
\text { glucose, insulin }\end{array}$ & NR/healthy & 52 & $180 / 122$ & $\begin{array}{l}\text { Enhanced fitness intervention/ } \\
\text { NR/No }\end{array}$ \\
\hline Miyaki (2012) (26) & 60 & NR & Female & & Sedentary/healthy & NR & $11 / 11$ & $\begin{array}{l}\text { Walking and cycling/moderate/ } \\
\text { No }\end{array}$ \\
\hline Kokkinos et al (27) & $(35-76)$ & $\begin{array}{c}\text { (T: } 30 \pm 4, C: \\
31 \pm 5)\end{array}$ & Male & $\begin{array}{c}\mathrm{TG}, \mathrm{HDL}, \mathrm{LDL}-\mathrm{C}, \\
\mathrm{TC}\end{array}$ & $\begin{array}{c}\text { Sedentary/ } \\
\text { hypertension }\end{array}$ & 16 & $15 / 19$ & Aerobic/exercise/moderate/No \\
\hline Karstoft et al (28) & $\begin{array}{c}(\mathrm{T}: 60.8+2.2, \mathrm{C}: \\
57.1 \pm 3.0)\end{array}$ & $N R$ & $\begin{array}{l}\text { Male and } \\
\text { female }\end{array}$ & $\begin{array}{l}\text { TG, HDL-C, } \\
\text { LDL-C, TC }\end{array}$ & $\begin{array}{l}\text { NR/diabetes } \\
\text { mellitus }\end{array}$ & 16 & $12 / 8$ & Walking/moderate/No \\
\hline $\begin{array}{l}\text { Kadoglou et } \\
\text { al(29) }\end{array}$ & $(64.1 \pm 9.2)$ & $\begin{array}{c}(\mathrm{T}: \\
32.74 \pm 4.05 \\
\mathrm{C}: 31.5 \pm 5.71)\end{array}$ & $\begin{array}{l}\text { Male and } \\
\text { female }\end{array}$ & $\begin{array}{c}\text { HDL C, LDL } \\
\text { C, TG, TC, } \\
\text { Fasting glucose, } \\
\text { Insulin, HOMA- } \\
\text { IR, HbA1C, } \\
\text { Fibrinogen }\end{array}$ & $\begin{array}{l}\text { NR/diabetes } \\
\text { mellitus }\end{array}$ & 12 & $23 / 24$ & $\begin{array}{l}\text { Resistance exercise/vigorous/ } \\
\text { No }\end{array}$ \\
\hline $\begin{array}{l}\text { Ghamarchehreh } \\
\text { et al (30) }\end{array}$ & $\begin{array}{c}(\mathrm{T}: 52.5 \pm 7.1, \mathrm{C}: \\
54.4 \pm 6.6)\end{array}$ & NR & $\begin{array}{l}\text { Male and } \\
\text { female }\end{array}$ & LDL, HDL, TG & Sedentary & 8 & $13 / 13$ & $\begin{array}{l}\text { Three } 45 \text {-min sittings per week } \\
\text { at } 55 \%-75 \% \text { of heart rate } \\
\text { reserve. RG participated in an } \\
8 \text { week }\end{array}$ \\
\hline $\begin{array}{l}\text { Ghamarchehreh } \\
\text { et al (30) }\end{array}$ & $\begin{array}{l}\text { (T: } 51.5 \pm 3.5, \mathrm{C}: \\
54.4 \pm 6.7)\end{array}$ & NR & $\begin{array}{l}\text { Male and } \\
\text { female }\end{array}$ & LDL, HDL, TG & Sedentary & 8 & $13 / 13$ & $\begin{array}{l}\text { resistance training, three } 45 \text { - } \\
\text { min sittings per week at } 50 \text { - } \\
70 \% \text { of } 1 \text { RM }\end{array}$ \\
\hline Finucane et al (31) & $(67.4-76.3)$ & 27.2 & $\begin{array}{l}\text { Male and } \\
\text { female }\end{array}$ & $\begin{array}{l}\text { HDL C, TG, LDL } \\
\text { C, HbA1c Fasting } \\
\text { glucose, TC }\end{array}$ & NR/healthy & 12 & $48 / 48$ & Cycle ergometer/moderate/No \\
\hline Fahlman et al (32) & $(74 \pm 5)$ & NR & Female only & $\begin{array}{l}\text { TC, HDL-C, } \\
\text { LDL-C, TG, TC/ } \\
\text { HDL-C }\end{array}$ & Sedentary/healthy & 10 & $15 / 15$ & $\begin{array}{l}10 \text { week of walking, } 3 \text { days/ } \\
\text { week, } 20 \text { until } 50 \mathrm{~min} / \mathrm{session} \text {, } \\
70 \% \text { of HRR }\end{array}$ \\
\hline Dalleck et al (33) & $\begin{array}{c}(\mathrm{T}: 55.4 \pm 3.2, \mathrm{C}: \\
57.4 \pm 4.6)\end{array}$ & Normal & Female only & $\begin{array}{l}\text { TG, HDL C, LDL } \\
\text { C, TC, Fasting } \\
\text { glucose }\end{array}$ & Sedentary/healthy & 12 & $8 / 10$ & $\mathrm{NR} /$ moderate/No \\
\hline $\begin{array}{l}\text { Boardley et al } \\
(34)\end{array}$ & over 65 & NR & $\begin{array}{l}\text { Male and } \\
\text { female }\end{array}$ & $\begin{array}{l}\text { TC, TG, HDL-C, } \\
\text { LDL-C }\end{array}$ & Sedentary/healthy & 16 & $33 / 35$ & $\begin{array}{l}\text { Resistance training and aerobic } \\
\text { walking/moderate/No }\end{array}$ \\
\hline Balducci et al (35) & $\begin{array}{c}(\mathrm{C}: 58.8 \pm 8.6, \mathrm{~T}: \\
58.8 \pm 8.5)\end{array}$ & $\begin{array}{l}(\mathrm{T}: 31.2 \pm 4.6 \\
\mathrm{C}: 31.6 \pm 4.7)\end{array}$ & $\begin{array}{l}\text { Male and } \\
\text { female }\end{array}$ & $\begin{array}{l}\text { HDL-C, LDL-C, } \\
\text { TC, TG, IR }\end{array}$ & $\begin{array}{c}\text { Sedentary/ } \\
\text { diabetes mellitus }\end{array}$ & 52 & $288 / 275$ & $\begin{array}{l}\text { Aerobic and resistance } \\
\text { training/moderate/No }\end{array}$ \\
\hline Baker et al (36) & 58.2 & NR & Male & $\begin{array}{c}\text { TC, HDL-C, } \\
\text { LDL-C, VLDL-C }\end{array}$ & Sedentary/healthy & 20 & $20 / 14$ & Aerobic training/vigorous/No \\
\hline Bobeuf et al (37) & $(59-73)$ & $26.2 \pm 2.6$ & $\begin{array}{l}\text { Male and } \\
\text { female }\end{array}$ & $\begin{array}{l}\text { HDL C, TC, TG, } \\
\text { LDL-C }\end{array}$ & Sedentary/healthy & 24 & $17 / 12$ & $\begin{array}{l}\text { Resistance training/vigorous/ } \\
\text { vitamins C/E supplementation }\end{array}$ \\
\hline Sung et al (38) & Over 70 & $N R$ & Female & $\begin{array}{l}\text { HDL C, LDL C, } \\
\text { TC, TG fasting } \\
\text { glucose, HbA1c }\end{array}$ & $\begin{array}{l}\text { NR/diabetes } \\
\text { mellitus }\end{array}$ & 24 & $22 / 18$ & Walking/moderate/No \\
\hline
\end{tabular}

T: training group; C: control group; NR: not reported; HDL C: high-density lipoprotein cholesterol: LDL C: low-density lipoprotein cholesterol; BMI: body mass index; HbA1c: glycosylated hemoglobin A1c; TC: total cholesterol; TG: triglyceride; IL: interleukin, HOMA IR: homeostatic model assessment insulin resistance 


\section{Influence of training on TG}

The present meta-analysis shows that TG levels significantly decreased by training (Figure 5$)(\mathrm{MD}=-0.62 ; P<0.001$, $\mathrm{SE}=0.12, \mathrm{~V}=0.01,95 \% \mathrm{CI}=-0.86-0.38, \mathrm{Z}=-5.03)$, and heterogeneity of the study was 33\%. In subgroups, aerobic training significantly decreased TG levels $(\mathrm{MD}=-0.73$; $P=0.001, \mathrm{SE}=0.16, \mathrm{~V}=0.02,95 \% \mathrm{CI}=-1.04-0.43, \mathrm{Z}$ $=-4.73)$, and resistance training significantly altered TG levels $(\mathrm{MD}=-0.36 ; P=0.07, \mathrm{SE}=0.2, \mathrm{~V}=0.04,95 \% \mathrm{CI}$ $=-0.760 .03, Z=-1.83)$.

\section{Subgroup Analysis}

Subgroup analysis was performed for different training methods (Figure 6). In subgroups, study heterogeneity remained high. HDL levels increased and LDL, TC, and TG decreased in subgroup analysis.

\section{Discussion}

In this systematic review and a meta-analysis, the effect of training on the lipid profile in elderly people was investigated. According to meta-analyses, regular training resulted in changes in the levels of HDL-C $(0.4 \mathrm{mg} / \mathrm{dL})$, LDL-C (-0.18 mg/dL), TC (-0.33 mg/dL), and TG (- 0.33 $\mathrm{mg} / \mathrm{dL}$ ). Furthermore, in the analysis of the subgroups, the resistance training produced changes in the levels of HDL-C (0.84 mg/dL), LDL-C (- $0.23 \mathrm{mg} / \mathrm{dL})$, TC (-0.32 $\mathrm{mg} / \mathrm{dL})$, and TG $(-1.3 \mathrm{mg} / \mathrm{dL})$. Several objective analyses have shown changes in lipid levels (39-41). Previous metaanalyses also suggested that training period or capacity could be correlated with improvement in lipid profile (40-42). For example, a meta-analysis indicated that each 10 -minute increase in exercise duration corresponded to an increase of $1.4 \mathrm{mg} / \mathrm{dL}$ in HDL level. The mechanisms by which training has improved lipid profile levels are not yet clear. However, since adipocytokines are believed to cause dyslipidemia (43), developments in lipid metabolism are seemingly associated with changes in adipocytokine levels. A large-scale randomized controlled trial described that both HDL-C and adiponectin levels increased by intensive lifestyle intervention and that changes in HDL-C levels were associated with changes in adiponectin levels (44). A current meta-analysis also indicated that resistance training reduced leptin levels and improved adiponectin levels (45); therefore, resistance training can improve lipid profile through changes in adipocytokine levels. A metaanalysis suggested that training and drug interventions are potentially similar in preventing CVD (46). Since the lipid profile is a risk factor for CVDs (47) and regular training improves lipid and lipoprotein levels (48), improvement in lipid levels can prevent CVD. It is believed the mechanism by which workout prevents cardiovascular disorder improves vascular function. A meta-analysis confirmed that significantly elevated the pulse wave velocity (49). A cross-sectional study revealed that pulse wave velocity is correlated with HDL-C, CT, and TG values (50). Furthermore, a controlled clinical study that covered confirmed that the percentage change in LDL-C or CT all through surgical procedure was associated with the percentage change in heart rate throughout surgical operation (51). A randomized controlled trial, which has been covered in the current meta-analysis, examined the associated vascular function and confirmed that swimming training significantly improved carotid artery compliance (52). The current meta-analysis had several limitations. The limitations of this study were as follows: some studies have very small study groups which tend to have more serious effects. The design of educational interventions varies in duration and intensity. The analyzed population was heterogeneous in terms of clinical condition, age, publication bias. Further RCTs are required to investigate the effect of aerobic training on individuals with specific disease states using different practice times and frequencies (number of exercise sessions per week) to determine the frequency of practice and appropriate duration for specific disease cases.

\section{Conclusion}

Although the present meta-analysis and systematic review suggest that training represents a suitable strategy to improve lipid profiles in the elderly, a cautious

\section{Group by}

\section{Subgroup training type}

advice training

Aerobic training

combined training

Resistance training

Overall
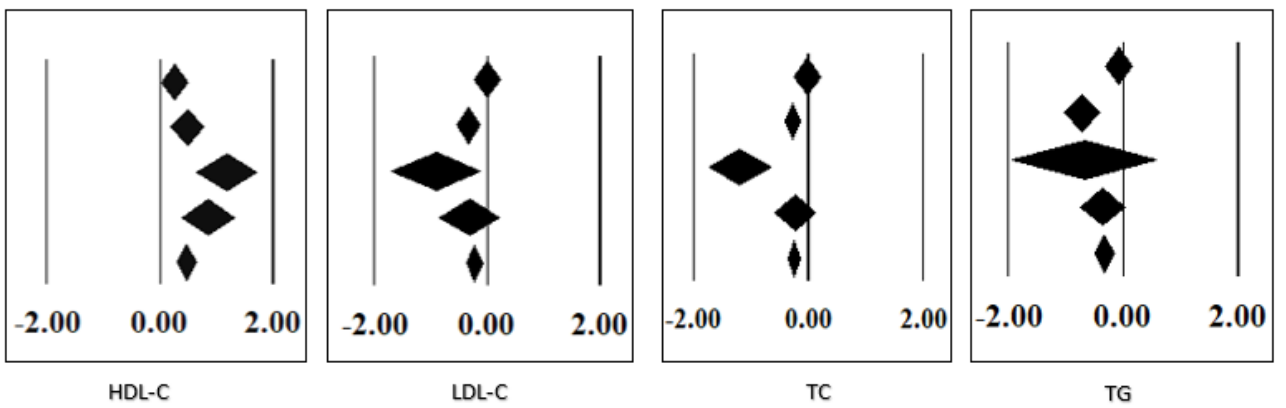

Figure 6. Effects of different training methods on HDL-C, LDL-C, TG, and TC Levels (mg/dL). Forest plot demonstrates mean differences (Cl). 
interpretation is warranted.

\section{Acknowledgments}

The authors would like to thanks the Vice Chancellor for Research and Technology of Shahrekord University.

Authors' Contributions

Article editing: MG. Collecting information, writing preliminary proposals: MF, TB, and MF.

\section{Conflict of Interests}

The author declares that they have no conflict of interests.

\section{Ethical Considerations}

In writing the article, the principles of publishing ethics have been observed.

\section{Funding/Support}

This study was not funded by a specific project grant.

\section{References}

1. Félix-Redondo FJ, Grau M, Fernández-Bergés D. Cholesterol and cardiovascular disease in the elderly. Facts and gaps. Aging Dis. 2013;4(3):154-69.

2. Grandjean PW, Crouse SF, Rohack JJ. Influence of cholesterol status on blood lipid and lipoprotein enzyme responses to aerobic exercise. J Appl Physiol (1985). 2000;89(2):472-80. doi: 10.1152/jappl.2000.89.2.472.

3. Meilhac O, Ramachandran S, Chiang K, Santanam N, Parthasarathy S. Role of arterial wall antioxidant defense in beneficial effects of exercise on atherosclerosis in mice. Arterioscler Thromb Vasc Biol. 2001;21(10):1681-8. doi: 10.1161/hq1001.097106.

4. Abuja PM, Albertini R. Methods for monitoring oxidative stress, lipid peroxidation and oxidation resistance of lipoproteins. Clin Chim Acta. 2001;306(1-2):1-17. doi: 10.1016/s00098981(01)00393-x.

5. Doi K, Sawada F, Toda G, Yamachika S, Seto S, Urata Y, et al. Alteration of antioxidants during the progression of heart disease in streptozotocin-induced diabetic rats. Free Radic Res. 2001;34(3):251-61. doi: 10.1080/10715760100300231.

6. Lin X, Zhang X, Guo J, Roberts CK, McKenzie S, Wu WC, et al. Effects of Exercise Training on Cardiorespiratory Fitness and Biomarkers of Cardiometabolic Health: A Systematic Review and Meta-Analysis of Randomized Controlled Trials. J Am Heart Assoc. 2015;4(7). doi: 10.1161/jaha.115.002014.

7. Ahn N, Kim K. High-density lipoprotein cholesterol (HDL-C) in cardiovascular disease: effect of exercise training. Integr Med Res. 2016;5(3):212-5. doi: 10.1016/j.imr.2016.07.001.

8. Ference BA, Ginsberg HN, Graham I, Ray KK, Packard C], Bruckert E, et al. Low-density lipoproteins cause atherosclerotic cardiovascular disease. 1. Evidence from genetic, epidemiologic, and clinical studies. A consensus statement from the European Atherosclerosis Society Consensus Panel. Eur Heart J. 2017;38(32):2459-72. doi: 10.1093/eurheartj/ehx144.

9. Zhang Y, Yang J, Ye J, Guo Q, Wang W, Sun Y, et al. Separate and combined associations of physical activity and obesity with lipid-related indices in non-diabetic and diabetic patients. Lipids Health Dis. 2019;18(1):49. doi: 10.1186/ s12944-019-0987-6.

10. Creighton BC, Hyde PN, Maresh CM, Kraemer WJ, Phinney SD, Volek JS. Paradox of hypercholesterolaemia in highly trained, keto-adapted athletes. BMJ Open Sport Exerc Med. 2018;4(1):e000429. doi: 10.1136/bmjsem-2018-000429.

11. Goldberg L, Elliot DL. The effect of physical activity on lipid and lipoprotein levels. Med Clin North Am. 1985;69(1):41-55.

12. Haskell WL. The influence of exercise training on plasma lipids and lipoproteins in health and disease. Acta Med Scand Suppl. 1986;711:25-37. doi: 10.1111/j.0954-6820.1986.tb08929.x.

13. Tran ZV, Weltman A, Glass GV, Mood DP. The effects of exercise on blood lipids and lipoproteins: a meta-analysis of studies. Med Sci Sports Exerc. 1983;15(5):393-402.

14. Higgins JP, Thompson SG, Deeks JJ, Altman DG. Measuring inconsistency in meta-analyses. BMJ. 2003;327(7414):55760. doi: 10.1136/bmj.327.7414.557.

15. Sutton AJ, Higgins JP. Recent developments in meta-analysis. Stat Med. 2008;27(5):625-50. doi: 10.1002/sim.2934.

16. Harbord RM, Higgins JPT. Meta-regression in Stata. Stata J. 2008;8(4):493-519. doi: 10.1177/1536867x0800800403.

17. Schünemann HJ, Oxman AD, Higgins JP, Vist GE, Glasziou P, Guyatt GH. Presenting results and 'Summary of findings' tables. In: Higgins JP, Green S, eds. Cochrane Handbook for Systematic Reviews of Interventions. Oxforrd: Cochrane Collaboration; 2008. doi: 10.1002/9780470712184.ch11.

18. Yoshizawa M, Maeda S, Miyaki A, Misono M, Choi Y, Shimojo $\mathrm{N}$, et al. Additive beneficial effects of lactotripeptides and aerobic exercise on arterial compliance in postmenopausal women. Am J Physiol Heart Circ Physiol. 2009;297(5):H1899903. doi: 10.1152/ajpheart.00433.2009.

19. Takeshima N, Rogers ME, Islam MM, Yamauchi T, Watanabe E, Okada A. Effect of concurrent aerobic and resistance circuit exercise training on fitness in older adults. Eur J Appl Physiol. 2004;93(1-2):173-82. doi: 10.1007/s00421-004-1193-3.

20. Takeshima N, Rogers ME, Watanabe E, Brechue WF, Okada A, Yamada T, et al. Water-based exercise improves health-related aspects of fitness in older women. Med Sci Sports Exerc. 2002;34(3):544-51. doi: 10.1097/00005768-20020300000024.

21. Nieman DC, Warren BJ, O'Donnell KA, Dotson RG, Butterworth DE, Henson DA. Physical activity and serum lipids and lipoproteins in elderly women. J Am Geriatr Soc. 1993;41(12):1339-44. doi: 10.1111/j.1532-5415.1993. tb06485.x.

22. Niederseer D, Ledl-Kurkowski E, Kvita K, Patsch W, Dela F, Mueller E, et al. Salzburg Skiing for the Elderly Study: changes in cardiovascular risk factors through skiing in the elderly. Scand J Med Sci Sports. 2011;21 Suppl 1:47-55. doi: 10.1111/j.1600-0838.2011.01341.x.

23. Nicklas BJ, Wang X, You T, Lyles MF, Demons J, Easter L, et al. Effect of exercise intensity on abdominal fat loss during calorie restriction in overweight and obese postmenopausal women: a randomized, controlled trial. Am J Clin Nutr. 2009;89(4):1043-52. doi: 10.3945/ajcn.2008.26938.

24. Morgan AL, Tobar DA, Snyder L. Walking toward a new me: the impact of prescribed walking 10,000 steps/day on physical and psychological well-being. J Phys Act Health. 2010;7(3):299-307. doi: 10.1123/jpah.7.3.299.

25. Morey MC, Pieper CF, Edelman DE, Yancy WS Jr, Green JB, Lum $\mathrm{H}$, et al. Enhanced fitness: a randomized controlled trial of the effects of home-based physical activity counseling on glycemic control in older adults with prediabetes mellitus. J Am Geriatr Soc. 2012;60(9):1655-62. doi: 10.1111/j.15325415.2012.04119.x.

26. Miyaki A, Maeda S, Choi Y, Akazawa N, Tanabe Y, Ajisaka R. Habitual aerobic exercise increases plasma pentraxin 3 levels in middle-aged and elderly women. Applied Physiology, Nutrition, and Metabolism 2012; 37(5): 907-911.

27. Kokkinos PF, Narayan P, Colleran J, Fletcher RD, Lakshman 
R, Papademetriou V. Effects of moderate intensity exercise on serum lipids in African-American men with severe systemic hypertension. Am J Cardiol. 1998;81(6):732-5. doi: 10.1016/ s0002-9149(97)01020-5.

28. Karstoft K, Winding K, Knudsen SH, Nielsen JS, Thomsen C, Pedersen BK, et al. The effects of free-living interval-walking training on glycemic control, body composition, and physical fitness in type 2 diabetic patients: a randomized, controlled trial. Diabetes Care. 2013;36(2):228-36. doi: 10.2337/dc120658.

29. Kadoglou NP, Vrabas IS, Kapelouzou A, Angelopoulou N. The association of physical activity with novel adipokines in patients with type 2 diabetes. Eur J Intern Med. 2012;23(2):13742. doi: 10.1016/j.ejim.2011.10.020.

30. Ghamarchehreh ME, Shamsoddini A, Alavian SM. Investigating the impact of eight weeks of aerobic and resistance training on blood lipid profile in elderly with non-alcoholic fatty liver disease: a randomized clinical trial. Gastroenterol Hepatol Bed Bench. 2019;12(3):190-6

31. Finucane FM, Sharp SJ, Purslow LR, Horton K, Horton J, Savage DB, et al. The effects of aerobic exercise on metabolic risk, insulin sensitivity and intrahepatic lipid in healthy older people from the Hertfordshire Cohort Study: a randomised controlled trial. Diabetologia. 2010;53(4):624-31. doi: 10.1007/s00125-009-1641-z.

32. Fahlman MM, Boardley D, Lambert CP, Flynn MG. Effects of endurance training and resistance training on plasma lipoprotein profiles in elderly women. J Gerontol A Biol Sci Med Sci. 2002;57(2):B54-60. doi: 10.1093/gerona/57.2.b54.

33. Dalleck LC, Allen BA, Hanson BA, Borresen EC, Erickson ME, De Lap SL. Dose-response relationship between moderateintensity exercise duration and coronary heart disease risk factors in postmenopausal women. J Womens Health (Larchmt). 2009;18(1):105-13. doi: 10.1089/jwh.2008.0790.

34. Boardley D, Fahlman M, Topp R, Morgan AL, McNevin N. The impact of exercise training on blood lipids in older adults. Am J Geriatr Cardiol. 2007;16(1):30-5. doi: 10.1111/j.10767460.2007.05353.x.

35. Balducci S, Zanuso S, Nicolucci A, De Feo P, Cavallo S, Cardelli $\mathrm{P}$, et al. Effect of an intensive exercise intervention strategy on modifiable cardiovascular risk factors in subjects with type 2 diabetes mellitus: a randomized controlled trial: the Italian Diabetes and Exercise Study (IDES). Arch Intern Med. 2010;170(20):1794-803. doi: 10.1001/ archinternmed.2010.380.

36. Baker TT, Allen D, Lei KY, Willcox KK. Alterations in lipid and protein profiles of plasma lipoproteins in middle-aged men consequent to an aerobic exercise program. Metabolism. 1986;35(11):1037-43. doi: 10.1016/0026-0495(86)90040-5.

37. Bobeuf F, Labonte M, Dionne IJ, Khalil A. Combined effect of antioxidant supplementation and resistance training on oxidative stress markers, muscle and body composition in an elderly population. J Nutr Health Aging. 2011;15(10):883-9. doi: 10.1007/s12603-011-0097-2.

38. Sung K, Bae S. Effects of a regular walking exercise program on behavioral and biochemical aspects in elderly people with type II diabetes. Nurs Health Sci. 2012;14(4):438-45. doi: 10.1111/j.1442-2018.2012.00690.x.

39. Igarashi Y, Nogami Y. Response of lipids and lipoproteins to regular aquatic endurance exercise: a meta-analysis of randomized controlled trials. J Atheroscler Thromb. 2019;26(1):14-30. doi: 10.5551/jat.42937.
40. Kelley GA, Kelley KS, Roberts S, Haskell W. Combined effects of aerobic exercise and diet on lipids and lipoproteins in overweight and obese adults: a meta-analysis. J Obes. 2012;2012:985902. doi: 10.1155/2012/985902.

41. Leon AS, Sanchez OA. Response of blood lipids to exercise training alone or combined with dietary intervention. Med Sci Sports Exerc. 2001;33(6 Suppl):S502-15. doi: 10.1097/00005768-200106001-00021.

42. Kodama S, Tanaka S, Saito K, Shu M, Sone Y, Onitake F, et al. Effect of aerobic exercise training on serum levels of high-density lipoprotein cholesterol: a meta-analysis. Arch Intern Med. 2007;167(10):999-1008. doi: 10.1001/ archinte.167.10.999.

43. Izadi V, Farabad E, Azadbakht L. Epidemiologic evidence on serum adiponectin level and lipid profile. Int J Prev Med. 2013;4(2):133-40.

44. Belalcazar LM, Lang W, Haffner SM, Hoogeveen RC, PiSunyer FX, Schwenke DC, et al. Adiponectin and the mediation of HDL-cholesterol change with improved lifestyle: the Look AHEAD Study. J Lipid Res. 2012;53(12):2726-33. doi: 10.1194/jlr.M030213.

45. Yu N, Ruan Y, Gao X, Sun J. Systematic review and metaanalysis of randomized, controlled trials on the effect of exercise on serum leptin and adiponectin in overweight and obese individuals. Horm Metab Res. 2017;49(3):164-73. doi: 10.1055/s-0042-121605.

46. Naci $H$, loannidis JP. Comparative effectiveness of exercise and drug interventions on mortality outcomes: metaepidemiological study. BMJ. 2013;347:f5577. doi: 10.1136/bmj.f5577.

47. Goff DC Jr, Lloyd-Jones DM, Bennett G, Coady S, D'Agostino RB Sr, Gibbons R, et al. 2013 ACC/AHA guideline on the assessment of cardiovascular risk: a report of the American College of Cardiology/American Heart Association Task Force on Practice Guidelines. J Am Coll Cardiol. 2014;63(25 Pt B):2935-59. doi: 10.1016/j.jacc.2013.11.005.

48. Chu P, Gotink RA, Yeh GY, Goldie SJ, Hunink MG. The effectiveness of yoga in modifying risk factors for cardiovascular disease and metabolic syndrome: a systematic review and metaanalysis of randomized controlled trials. Eur J Prev Cardiol. 2016;23(3):291-307. doi: 10.1177/2047487314562741.

49. Ashor AW, Lara J, Siervo M, Celis-Morales C, Mathers JC. Effects of exercise modalities on arterial stiffness and wave reflection: a systematic review and meta-analysis of randomized controlled trials. PLoS One. 2014;9(10):e110034. doi: 10.1371/journal.pone.0110034.

50. Tomiyama H, Yamashina A, Arai T, Hirose K, Koji Y, Chikamori $\mathrm{T}$, et al. Influences of age and gender on results of noninvasive brachial-ankle pulse wave velocity measurement--a survey of 12517 subjects. Atherosclerosis. 2003;166(2):303-9. doi: 10.1016/s0021-9150(02)00332-5.

51. Kawasaki T, Sullivan CV, Ozoe N, Higaki H, Kawasaki J. A long-term, comprehensive exercise program that incorporates a variety of physical activities improved the blood pressure, lipid and glucose metabolism, arterial stiffness, and balance of middle-aged and elderly Japanese. Hypertens Res. 2011;34(9):1059-66. doi: 10.1038/hr.2011.81.

52. Nualnim N, Parkhurst K, Dhindsa M, Tarumi T, Vavrek J, Tanaka H. Effects of swimming training on blood pressure and vascular function in adults $>50$ years of age. Am J Cardiol. 2012;109(7):1005-10. doi: 10.1016/j.amjcard.2011.11.029. 